The AsTROPHYSICAL JOURNAL, 434: 192-199, 1994 October 10

(C) 1994. The American Astronomical Society. All rights reserved. Printed in U.S.A

\title{
STEADY STATE MAGNETIC RECONNECTION IN PLANAR GEOMETRIES
}

\author{
I. J. D. Craig and S. M. Henton \\ University of Waikato, Private Bag 3105, Hamilton, New Zealand \\ Received 1993 December 28; accepted 1994 March 30
}

\begin{abstract}
The problem of nonlinear, steady state magnetic reconnection in incompressible plasmas is considered. A self-consistent treatment is developed which allows a formal analytic solution of the resistive induction equation, valid for all sub-Alfvénic velocity fields. This solution relates the velocity stream function to the global magnetic field structure and highlights several key properties of the resistive system. In particular, the field lines are isobars of uniform current density and characteristics of the velocity stream function.

The impact of this analysis on "fast" magnetic reconnection is then considered. Despite strong constraints on the flow topology-no separatrix flows are allowed-fast, nonlinear models can be developed selfconsistently. It is shown that the reconnection rate is maintained against reductions in the plasma restrictivity by the increasing amplitude but decreasing width of the current layer aligned to the separatrix. It is concluded that "open" flow topologies allow fast reconnection to continue under conditions which lead to stalling in "closed" or periodic geometries.

Subject headings: MHD - plasmas
\end{abstract}

\section{INTRODUCTION}

Despite the vigorous development of steady state, magnetic reconnection theory over the last 30 years (e.g., Vasyliunas 1975; Forbes \& Priest 1987), a definitive global model of the magnetic merging process has yet to be established. A major difficulty with the traditional, semi-analytic approach is matching the large-scale advection of the outer magnetic field to a localized diffusion layer surrounding the neutral point. Recent numerical simulations of magnetic merging have shed some light on the global problem but often at the expense of traditional "fast" reconnection theory (e.g., Biskamp 1986). Whether the classical approach leads to a reconnective model which can account for, say, the explosive energy release in a solar flare, remains highly contentious.

More recently, the problem of dynamic reconnection has been addressed. An analytic treatment is possible for the case of small amplitude, compressible disturbances on $X$-type neutral points (Craig \& McClymont 1991; Hassam 1992). Remarkably, all global disturbances-not just perturbations altering the intrinsic $X$-point topology-dissipate resistively on a "fast" timescale, that is, over a timescale effectively independent of the plasma resistivity (Craig \& McClymont 1993). This analysis also presents a graphic description of the dynamic collapse to small length scales that must occur if reconnection is to provide a viable mechanism for fast magnetic annihilation (Craig \& Watson 1992). Numerical simulations confirm that fast, dynamic reconnection persists for large amplitude disturbances (Biskamp \& Welter 1980; DeLuca \& Craig 1992; Rickard \& Craig 1993) but the dissipation rate eventually stalls when finite compressibility and nonplanar magnetic field components are introduced (Craig, Henton, \& Rickard 1993). Apparently, the strong current localizations necessary for rapid ohmic dissipation are inhibited by the buildup of back-pressures at the neutral point.

To what extent the stalling of fast, dynamic reconnection is an artifact of the restricted flow topology imposed by the "closed" $X$-point geometry is unclear. But it seems likely that the "open" flow topology of traditional steady state theory, or more general three-dimensional geometries, could allow sufficient freedom to prevent strong pressure gradients retarding the flow. Motivated by these considerations, Craig \& Rickard (1994) demonstrate analytically that fast, linear, steady state reconnection in incompressible plasmas is maintained for the "open" planar geometries of traditional theory. However, the analytic solution for the flow topology explicitly forbids fluid motions across the separatrices, a result in contradiction to the flow topologies of traditional steady state theory!

The purpose of this paper is to extend the linear analysis of Craig \& Rickard (1994) into the nonlinear regime of the classical theory. This approach allows the diffusion and advection regions to be treated self-consistently in a mathematically unambiguous fashion. Specifically, an exact analytic solution of the resistive induction equation is constructed that relates the plasma flow to the magnetic field structure under very general conditions. This construction makes explicit certain fundamental properties of all resistive reconnective solutions. The main restriction on the analysis is that flow speeds are limited to below the Alfvén Mach number of the plasma-a constraint also shared by the traditional quasi-linear approach (Priest \& Forbes 1986).

The paper is organized as follows. In $\S 2$ we introduce the resistive system-and briefly background the conventional quasi-linear approach-before discussing the details and implications of the present analysis. Section 3 describes applications of the theory to the problem of "fast" linear and nonlinear magnetic reconnection. A summary of our findings is presented in $\S 4$.

\section{STEADY STATE RECONNECTION ANALYSIS}

\subsection{Resistive System}

We consider the steady state momentum and induction equations for a resistive, incompressible plasma in a twodimensional geometry. The reconnective system is conve- 
niently expressed in the nondimensional form,

$$
\begin{gathered}
(v \cdot \nabla) v=-\nabla^{2} \psi \nabla \psi-\nabla p, \\
v \cdot \nabla \psi=E+\eta \nabla^{2} \psi,
\end{gathered}
$$

where $p$ is the plasma pressure and $\psi$ is a flux function for the magnetic field

$$
\boldsymbol{B}=\boldsymbol{\nabla} \times \psi \hat{\boldsymbol{z}} .
$$

The incompressibility condition is satisfied by introducing a velocity stream function

$$
\boldsymbol{v}=\boldsymbol{\nabla} \times \phi \hat{\boldsymbol{z}},
$$

where $v=(u, v)=\left(\phi_{y},-\phi_{x}\right)$ and differentiation of the dependent variables is indicated by subscript notation.

We adopt the traditional symmetries, namely,

$$
u=\psi_{x}=0 \text { on } x=0, v=\psi_{y}=0 \quad \text { on } y=0 \text {, }
$$

and thus model the positive quadrant of the reconnection region $(0 \leq x, y \leq 1)$. The origin is both a neutral point of the magnetic field and a stagnation point of the plasma flow.

The system is specified by the dimensionless parameters $E$ and $\eta$. Here $E$ defines a uniform electric field which determines the strength of the external plasma flow. With flow speeds measured in units of the boundary Alfvén speed, the dimensionless resistivity $\eta$ is an inverse magnetic Reynolds number of order $10^{-10}$ for typical coronal plasmas. Consequently, to avoid "slow" magnetic merging, the reconnection rate cannot depend strongly upon $\eta$. This is tantamount to the assumption that, for well-defined "external" boundary conditions, $E$ can be chosen essentially independent of the plasma resistivity.

Before considering the reconnective system in more detail, it is instructive to outline the traditional quasi-linear approach, as systematized by Priest \& Forbes (1986).

\subsection{Perturbation Expansion Approach}

The classical approach assumes a perturbation expansion, in which the Alfvénic flow speed is the implicit small parameter, for an "outer field" region of zero resistivity:

$$
\psi=\psi_{0}+\psi_{1}+\ldots, \quad v=v_{0}+v_{1}+\ldots .
$$

The equilibrium structure comprises stationary $\left(v_{0}=0\right)$, antiparallel magnetic fields: however, the transition layer across the neutral line - a region of high current density - is never considered. The effects of finite resistivity are likewise ignored beyond the assumption that the dimensions of the current sheet can be adjusted to match the external flow solution. Under these restrictions, Priest \& Forbes (1986) show that the perturbation expansion leads to families of "fast" reconnection models, for example, Petschek (1964).

The problems associated with the traditional approach are well known and will not be detailed here. We simply mention that attempts to confirm the classical picture by direct simulation have been largely unsuccessful (as emphasized by Biskamp 1986) unless bolstered by additional effects not included in the original formulation of the problem (e.g., the nonuniform plasma resistivities of Yan et al. 1992). Interpretive problems are compounded by the notorious difficulty of adopting boundary conditions for "open" steady state flow (Forbes \& Priest 1987) and by the limited range of resistivities that can be simulated numerically.

\subsection{Momentum Equation for Sub-Alfvénic Flows}

In the following analysis we neglect the convective derivative in the momentum equation (2.1). This simplification only affects the second and higher order components of the momentum equation which remain small for sub-Alfvénic flow velocities.

In terms of the stream function (2.4) the system reduces to

$$
\begin{gathered}
\nabla^{2} \psi \nabla \psi=-\nabla p, \\
(\nabla \psi \times \nabla \phi) \cdot \hat{z}=E+\eta \nabla^{2} \psi .
\end{gathered}
$$

To satisfy equation (2.6) we must have $p=p(\psi)$ and so

$$
\nabla^{2} \psi=-p^{\prime}(\psi) \text {. }
$$

The magnetic field lines are then isobars along which the current density is constant. A further implication is that no plasma can flow across the separatrices. This follows by noting that since

$$
p^{\prime}(\psi)=-\nabla^{2} \psi=E / \eta,
$$

is satisfied at the origin, equation (2.9) holds along any magnetic field line that threads the neutral point. Therefore the flow term in the induction equation (2.7) must vanish on the separatrix.

Returning to the momentum equation, we note that equation (2.6) must contain the totality of information in the zerothand first-order components of the magnetic field. To be more explicit, we write the pressure as

$$
p(\psi)=p_{0}(\psi)+\Delta p(\psi)
$$

and set $\psi=\psi_{0}+\psi_{1}$. An expansion about $\psi_{0}$ then yields the equilibrium structure

$$
\nabla^{2} \psi_{0}=-p_{0}^{\prime}\left(\psi_{0}\right),
$$

and the first-order solution (Craig \& Rickard 1994)

$$
\nabla^{2} \psi_{1}+p_{0}^{\prime \prime}\left(\psi_{0}\right) \psi_{1}=-\Delta p^{\prime}\left(\psi_{0}\right) \text {. }
$$

The requirements for solving equations (2.11) and (2.12) are well known. Each source function must be specified within the region of interest, while a linear combination of the dependent variable and its normal derivative must be prescribed on the boundary. The equilibrium structure $(2.11)$ can be chosen current free, but the first-order solution must contain strong, localized currents at the neutral point if significant reconnection is to occur. The form of the source function $\Delta p^{\prime}\left(\psi_{0}\right)$ in the Helmholtz equation (2.12) is therefore critical in determining the reconnection speed. Similarly, for the full nonlinear equation (2.8), it is the increment function $\Delta p^{\prime}(\psi)$ that is of central importance.

\subsection{Analytic Solution of the Induction Equation}

In the present approach, the magnetic field is determined independently of the induction equation, mainly by the choice of the generating function $p^{\prime}(\psi)$. Although we cannot assert that the full nonlinear equation (2.8) always has well-posed solutions, we can guarantee solutions in linear (Craig \& Rickard 1994) and quasi-linear applications (Priest \& Forbes 1986).

Let us assume therefore that a solution for $\psi$ can be constructed. The induction equation (2.7) can be written as

$$
\phi_{y} \psi_{x}-\phi_{x} \psi_{y}=E-\eta p^{\prime}(\psi) \text {. }
$$

According to the method of characteristics, the stream function $\phi$ can be determined from the ordinary differential equations

$$
-\frac{d x}{\psi_{y}}=\frac{d y}{\psi_{x}}=\frac{d \phi}{E-\eta p^{\prime}(\psi)} .
$$


The characteristics are curves in the $x-y$ plane on which the total differential of $\phi$ is specified. These are simply the magnetic field lines given by

$$
d \psi=\psi_{x} d x+\psi_{y} d y=0 .
$$

We can express the solution for $\phi$ as a quadrature over either of the independent variables. Choosing $y$ to parameterize the characteristics gives the general solution

$$
\phi(x, y)=\left[E-\eta p^{\prime}(\psi)\right] \int^{y} \frac{d y}{\psi_{x}[x(\psi, y), y]}+\mu(\psi),
$$

where the arbitrary function $\mu(\psi)$ is the integration constant for each characteristic. Since $\nabla \mu=\mu^{\prime} \nabla \psi$, the velocity field $\nabla \mu \times \hat{z}$ is directed along the magnetic field lines.

We conclude that (2.15) is the general solution of the induction equation valid for sub-Alfvénic flows. The solution is completely determined when boundary conditions are specified on any noncharacteristic curve in the $x-y$ plane. For the standard reconnection geometry we can choose $\phi=0$ along the $x$ and $y$-axes and set $\mu(\psi)=0$ to eliminate plasma flow across the inner boundaries. Obviously, no freedom is left to specify " outer" boundary conditions for $\phi$.

\subsection{Universal Features of the Reconnection Problem}

We now detail several fundamental properties of the reconnective system. For convenience we shall normalize $\psi$ so the level $\psi=0$ defines the separatrices of the magnetic field. We then have

1. The magnetic field lines are isobars along which the current density is constant; the field lines are characteristics of the velocity stream function.

2. No separatrix flows are allowed (by eq. [2.15]). form

3. The pressure in the vicinity of the separatrix (2.9) has the

$$
p=p_{N}+\frac{E}{\eta} \psi
$$

where $p_{N}$ is some constant.

It should be emphasized that, although these results contradict traditional steady state models, they can only be undone by modifying the resistive system in some essential way. In particular, as regions of strong current contiguous with the separatrices extend uniformly from the origin to the outer boundary, it is incorrect to assume that high currents can be localized within a small "rectangular" diffusion region surrounding the neutral point. Admittedly, such localized current structures may be achieved by the expedient of nonuniform resistivities (e.g., Yan, Lee, \& Priest 1992), in much the same way as flow across the separatrices requires the inclusion of fluid viscosity (as in Priest et al. 1994). However, the motivation for introducing such complications seems dubious given that the simplest resistive systems are not well understood.

The question of whether solutions that disqualify fluid motion across the separatrices can be classed as "reconnective" is essentially a matter of definition (Priest et al. 1994). If a narrow definition is taken which requires separatrix flow (Vasyluinas 1975), then we have proved that "reconnection" is impossible for the invicid resistive system.
More seriously, this definition leads to the bizarre result that "reconnection" must depend on the level of the fluid viscosity in the problem. This seems absurd given that reconnection is intrinsically resistive, and capable of rigorous definition even for invicid plasmas (e.g., Craig \& McClymont 1993)!

Finally, we point out an interesting ramification of our analysis. Suppose equation (2.16) is applied to a magnetic field which is locally " $X$-type"; that is, locally of the form $\psi \propto\left(x^{2}-\alpha^{2} y^{2}\right), 0<\alpha \leq 1$. The origin is then a saddle point of the pressure being a maximum on the inflow axis and a minimum on the outflow axis.

\section{FAST RECONNECTIVE SOLUTIONS}

\subsection{Introduction}

We now investigate the possibility of fast steady state energy dissipation. As we have the freedom to specify the generating function $p^{\prime}(\psi)$ and boundary conditions (Neumann or Dirichlet) for $\psi$, we cannot uniquely determine a fast reconnection model. Our aim therefore is to demonstrate fast, linear and nonlinear solutions under simple, plausible parameterizations of the generating function.

So far we have considered only one measure of "fast" reconnection, namely, the flux annihilation rate at the neutral point as determined by the scaling of $E$ with $\eta$. Also of interest however, are the total current in the dissipation region and the ohmic release rate, namely

$$
I_{\eta}=\int_{S}\left|\nabla^{2} \psi\right| d x d y, \quad W_{\eta}=\eta \int_{S}\left|\nabla^{2} \psi\right|^{2} d x d y .
$$

In particular, $W_{\eta}$ measures the transfer of magnetic energy into heat, as opposed to the kinetic energy of mass motion. We follow DeLuca \& Craig (1992) in adopting "fast" to indicate that at least one of the measures, $E$ or $W_{\eta}$, scales independently of any positive power of $\eta$.

\subsection{The Form of the Generating Function}

Although the construction of fast reconnection mechanisms is a central goal of magnetic merging theory, it appears that only a fully dynamic model is capable of yielding unambiguous reconnection scalings. In the traditional steady state approach, for example, families of reconnection models are distinguished by the nature of the flow topology in the vicinity of the neutral point (Priest \& Forbes 1986). Each family differs with respect to the gross properties of the current sheet. In contrast a unique solution is guaranteed for any dynamic model that evolves from well-defined initial conditions.

Arguably, the simplest dynamic model for fast dissipation is provided by the theory of the linearized $X$-point (Craig \& McClymont 1993). In this case $I_{\eta}, W_{\eta}$, and the flux annihilation rate all scale independently of resistivity - at least to within nondimensional factors of $O(|\ln \eta|)$. The reconnection rate stalls (only $I_{\eta}$ remains invariant) when significant gas pressures are introduced, but it is conjectured that "open "flow topologies may allow fast reconnection to persist, even for incompressible plasmas (Craig \& Watson 1992).

Motivated by these considerations, we consider a simple analytic form for the generating function

$$
p^{\prime}(\psi)=\frac{E}{\eta} F(\lambda \psi), \quad \lambda=\beta \frac{E}{\eta},
$$


where $\beta$ is some fixed number of order unity. Considerations of symmetry imply that $F$ must be even and for analytic convenience we take $F(x)=\operatorname{sech}^{2}(x)$. The key point, as confirmed

I below, is that (3.2) naturally reproduces the scaling of the dynamic $X$-point theory while allowing plausible constructions into the nonlinear regime.

\subsection{Linear $X$-Point Solutions}

We now apply the analysis of $\S 2$ to equilibrium $X$-points of the form

$$
\psi_{0}=\frac{1}{2}\left(x^{2}-\alpha^{2} y^{2}\right), \quad 0<\alpha \leq 1 .
$$

The background current is uniform so equation (2.12) reduces to

$$
\nabla^{2} \psi_{1}=-\Delta p^{\prime}\left(\psi_{0}\right)=-\frac{E}{\eta} \operatorname{sech}^{2}\left(\lambda \psi_{0}\right) .
$$

The solution for the stream function below the separatrix $(x>\alpha y)$ is given by

$$
\phi=\frac{\left[E-\eta \Delta p^{\prime}\left(\psi_{0}\right)\right]}{\alpha} \sinh ^{-1}\left[\frac{\alpha y}{\left(2\left|\psi_{0}\right|\right)^{1 / 2}}\right] .
$$

Above the separatrix $(x<\alpha y)$, the solution is obtained by replacing $\alpha y$ with $x$ in the numerator of the hyperbolic argument.

Figures 1 and 2 show the basic features of the linear solution for the case $E=1, \eta=0.1, \beta=1$, and $\alpha=3^{1 / 2} / 2$. Contours of the equilibrium flux function $\left(\psi_{0}\right)$ and the velocity stream function $(\phi)$ confirm the presence of strong shearing flows parallel to the separatrices (Figs. $1 a$ and $1 b$, respectively). Plasma inflow along the negative $x$-axis is compensated by an outflow jet below the separatrix. Similarly, the strong inflow jet above the separatrix is balanced by a weaker, but more extended, outflow through the upper boundary.

Figure $2 a$ illustrates the ridge of peak current that extends uniformly along the separatrix to the outer boundary. Notably, the contours of the perturbed flux function in Figure $2 b$ resem- ble the aximuthal contours of dynamic $X$-point theory (Craig \& Watson 1992), although variations caused by extended current ridges along the separatrix are clearly apparent.

We now determine reconnective scalings under the assumption that $E$ is invariant with resistivity. First we note that length scales in the vicinity of the neutral point (3.4) are determined by $\lambda^{-1 / 2} \sim \eta^{1 / 2}$. Taking a localized current of magnitude $E / \eta$ over the area $\Delta S \sim \eta$ yields $I_{\eta} \sim W_{\eta} \sim \eta^{0}$. This simple dimensional argument-which ignores the current extension along the separatrices - already suggests that fast ohmic dissipation is achieved.

To obtain more precise scalings we introduce the variable change

$$
r=x-\alpha y, \quad s=x+\alpha y .
$$

The integral for the current then transforms to

$$
I_{\eta}=\frac{2}{\alpha} \int_{0}^{2 \alpha} \int_{0}^{2 \alpha} \frac{E}{\eta} \operatorname{sech}^{2}\left(\frac{\lambda r s}{2}\right) d r d s,
$$

and so

$$
I_{\eta}=\frac{4}{\alpha \beta} \int_{0}^{2 \lambda \alpha^{2}} \tanh (t) \frac{d t}{t}
$$

It follows that

$$
\frac{\alpha \beta}{4} I_{\eta}=B_{0}+B_{1} \ln \left(2 \lambda \alpha^{2}\right)
$$

where $B_{0}$ is a sum (of order unity) of Bernoulli numbers and $B_{1}$ satisfies $\tanh (1) \approx 0.762<B_{1}<1$. A similar evaluation of the ohmic dissipation rate gives the scaling

$$
W_{\eta} \sim I_{\eta} \sim|\ln \eta| \text {. }
$$

Since $E$ is invariant the model is marginally more effective at converting magnetic energy to heat than the kinetic energy of fluid motion. However, both reconnection measures are fast in agreement with dynamic $X$-point theory.

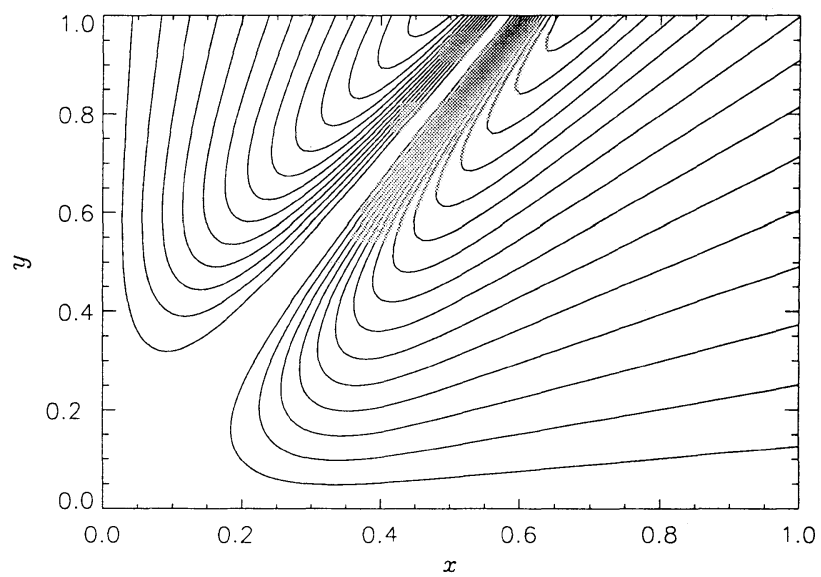

Fig. $1 b$

FIG. 1.-Plots of $\psi$ and $\phi$ for the linearized $X$-point problem with parameters $\eta=0.01, E=0.1, \alpha=\sqrt{3} / 2$, and $\beta=1$. The background magnetic field $\left(\psi_{0}\right)$ is characterised by a separatrix inclined at $60^{\circ}$ to the $x$-axis $(a)$. The stream function $(b)$ is evaluated from solution $(3.5)$ and exhibits strong shear-flows parallel to the separatrix. 


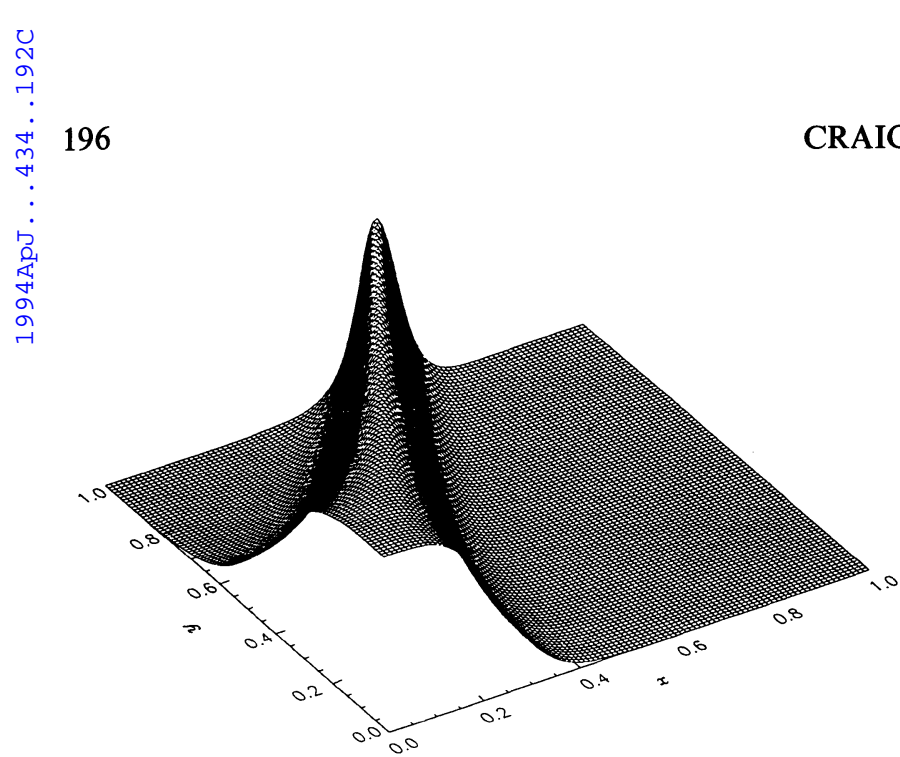

FIG. $2 a$

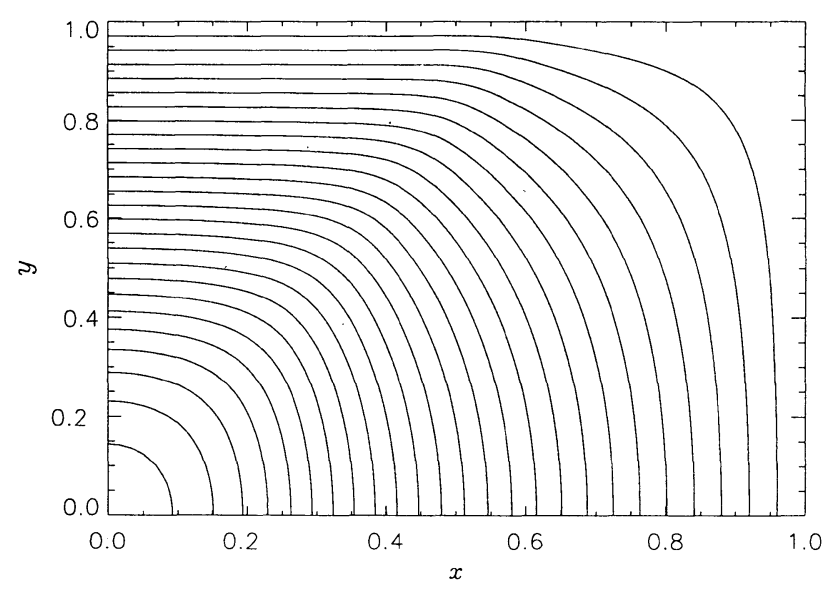

FIG. $2 b$

FIG. 2.-(a) Surface plot of the perturbation current for the linearized problem of Fig. 1. (b) Contours of the perturbed flux functions $\psi_{1}$.

Finally, we mention that the major limitation of the linearised model is the severe restriction $[O(\eta)]$ on the perturbation amplitude. If this is exceeded, the perturbation field (Fig. 2b) overwhelms the background field and the linearization fails (as discussed in Craig \& Watson 1992).

\subsection{Nonlinear Solutions}

For large amplitude displacements the momentum equation (2.8) is highly nonlinear and therefore difficult to treat analytically. In practice we employ an implicit relaxation technique to model the time-evolution equation

$$
\dot{\psi}=p^{\prime}(\psi)+\nabla^{2} \psi
$$

Boundary conditions are set by assuming initial conditions of the $X$-point form (3.3). After each iteration $\psi$ is implicitly normalized so that $\psi=0$ defines the separatrix contour. Once a relaxed solution for $\psi$ is achieved, it is a simple exercise to determine the stream function $\phi$, for instance by numerically integrating along the characteristics (2.14).

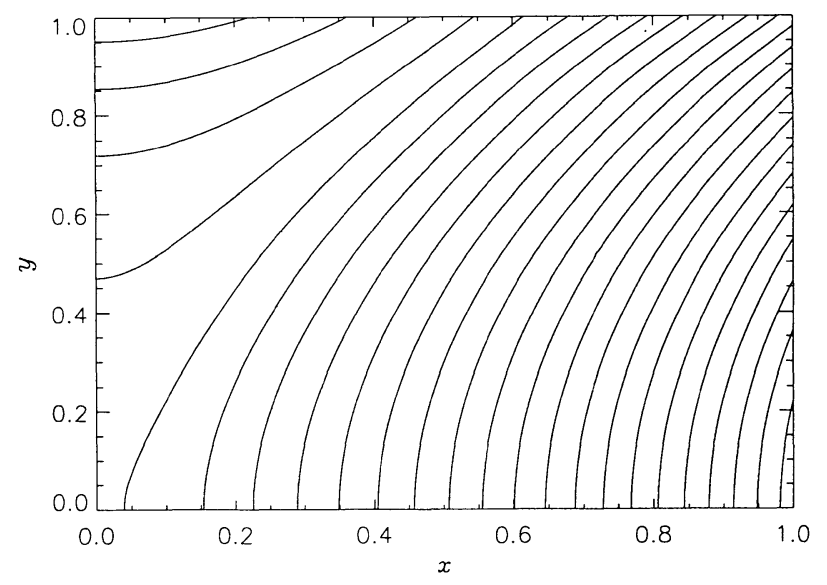

Fig. $3 a$
Results from a typical nonlinear computation are presented in Figure $3(\eta=0.01, E=0.1, \alpha=1$, and $\beta=8)$. In common with the linear analysis of $\S 3.3$, strong shear flows parallel to the separatrices are a salient feature (Fig. $3 b$ ). The nonlinear results however, as illustrated in Figure $4 a$, show a tendency for the separatrix structure near the neutral point to flatten into a quasi-one-dimensional current sheet. The saddle point pressure variation close to the origin is shown in Figure $4 b$.

To determine reconnective scalings we systematically reduce $\eta$ while keeping all other parameters fixed. Figure $5 a$ confirms that $I_{\eta}$ and $W_{\eta}$ remain essentially independent of resistivity. However, the increasing quasi-one-dimensionality of the current profile implies that the reconnection scalings are maintained predominantly by reductions in the width of the current sheet. Figure $5 b$ shows that the width of the sheet indeed reduces in proportion to $\eta$. As the length is effectively invariant, the area of the current sheet must scale as the resistivityhence reconnective scaling consistent with the linear model.

Finally, we illustrate the dramatic collapse of the current structure with reductions in resistivity. The contour plots of

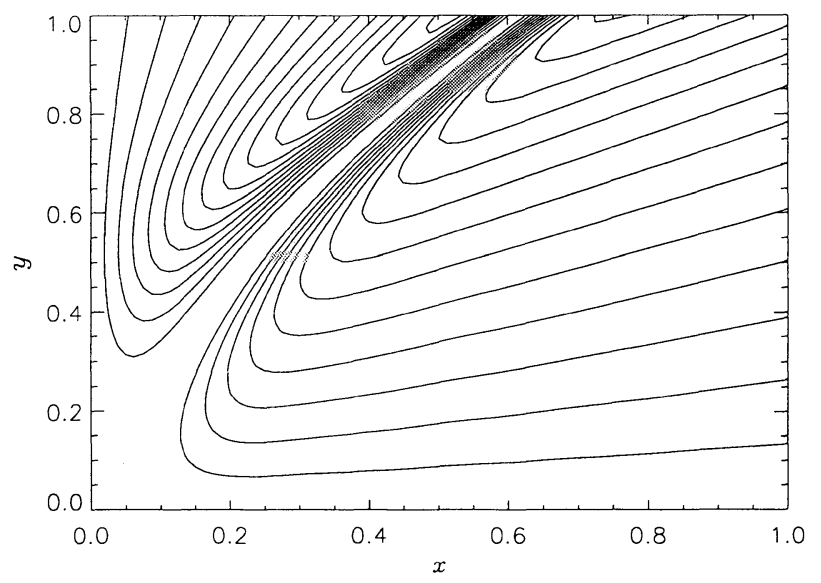

FIG. $3 b$

FIG. 3.-Plots of $\psi(a)$ and $\phi(b)$ for the nonlinear solution with parameters $\eta=0.01, E=0.1, \alpha=1$, and $\beta=8$. A notable feature is the flattening of the separatrix near the neutral point. 


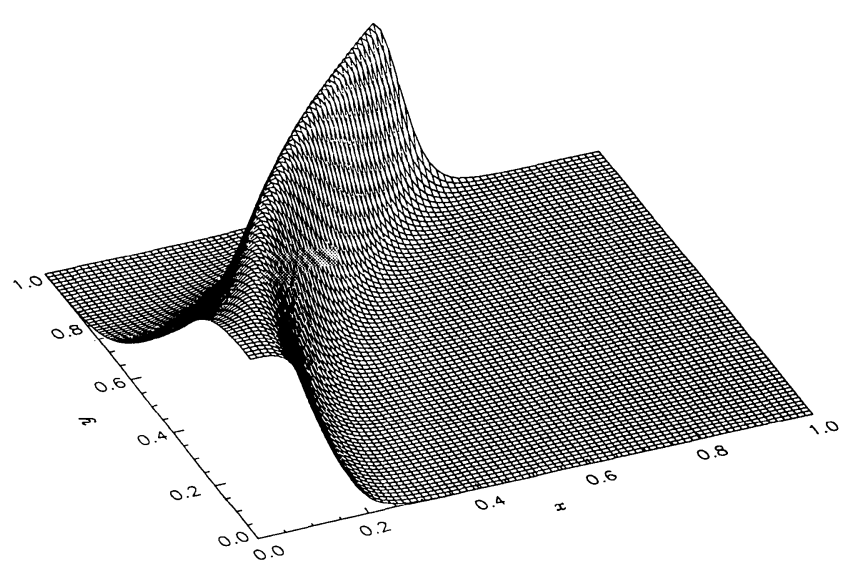

FIG. $4 a$

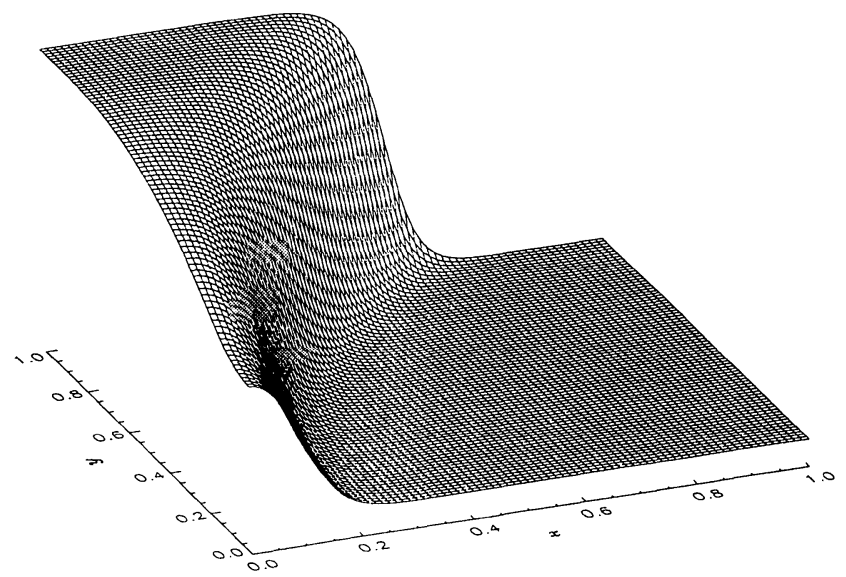

FIG. $4 b$

Fig. 4. - (a) Surface plot of the current density for the nonlinear solution of Fig. 3. (b) Surface plot of the pressure variation.

Figure 6 show the effect of reducing $\eta$ by a factor of 10 . Quasilinear structures obtained at large $\eta$ rapidly degenerate to thin current sheets aligned to the separatrix.

\subsection{A Solution for Antiparallel Magnetic Merging}

As a final demonstration of the fast reconnective scalings associated with equation (3.2), we consider the merging of
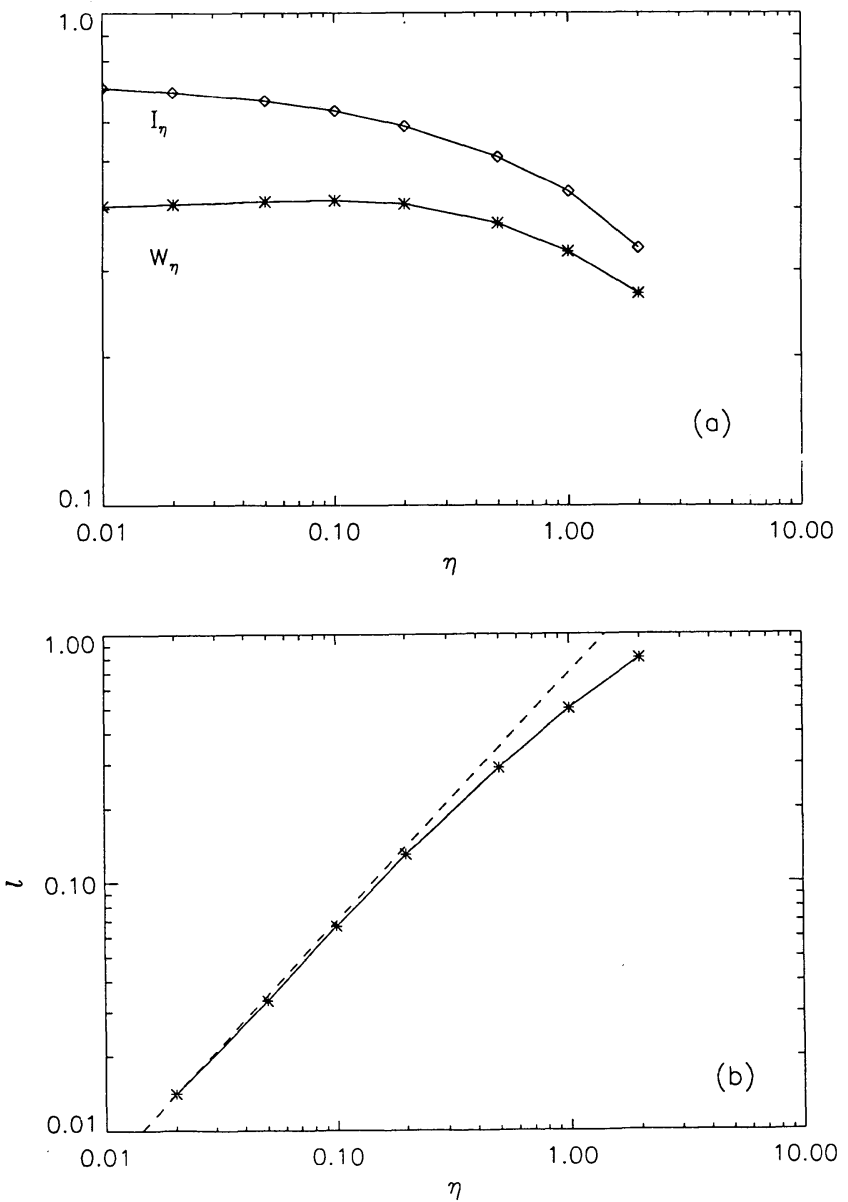

FIG. 5.- (a) Plot of integrated current and ohmic dissipation rate vs. resistivity. (b) Current sheet width $(l)$ vs. resistivity. The dashed line indicates the reference scaling $l \sim \eta^{1}$. strictly antiparallel magnetic fields. Taking $\psi=\psi(x)$ and specializing to $\beta=1$ in equation (3.2) gives

$$
\psi_{x}=\tanh \left(\frac{E}{\eta} x\right), \quad \phi=E y \psi_{x},
$$

where $\psi$ has the explicit form

$$
\psi(x)=\psi_{N}+\frac{\eta}{E} \ln \left[\cosh \left(\frac{E}{\eta} x\right)\right] .
$$

This simple example illustrates several key features of the problem. With $E$ fixed, the magnetic field and inflow speed on the outer boundary $(x=1)$ are invariant with $\eta$. Reductions in the plasma resistivity are compensated by the decreasing width $(\sim \eta)$ of the transition layer overlying the $y$-axis. A simple calculation confirms the invariance of the integrated current in the diffusion layer $I_{\eta}$ and the ohmic dissipation rate $W_{\eta}$. Notably, these scalings - and the width of the current layerare consistent with the nonlinear, two-dimensional field scalings of the previous section.

The shear-flow topology however, degenerates in the case of a strictly one-dimensional field. Close to the origin a stagnation point flow exists $\left(\phi \approx E^{2} x y / \eta\right)$ with plasma being ejected in a narrow jet along the neutral line. The restriction of subAlfvénic outflows can always be met, formally at least, by taking $E$ sufficiently small: specifically for $\eta>\eta_{\min }$ say, we take $E \simeq \eta_{\min }^{1 / 2}$. None the less, relatively large outflow speeds appear a natural feature of antiparallel merging.

\subsection{Summary}

The present results confirm that fast shear-flow solutions can be generated self-consistently in both linear and nonlinear applications. Although all models have essentially the same ohmic dissipation and flux annihilation scalings, the current structure in the vicinity of the neutral point degenerates from near-cylindrical to quasi-one-dimensional with the increasing nonlinearity of the solution. As a result, in the nonlinear models, reductions in resistivity are compensated entirely by the diminishing width $(\sim \eta)$ of the current layer. This contrasts with the linear models in which the bulk of the current is contained in a cylindrical spike (of area $\sim \eta$ ) surrounding the origin.

In practice, we note that the strong shear flows that characterize our solutions will be modified by viscous effects. 

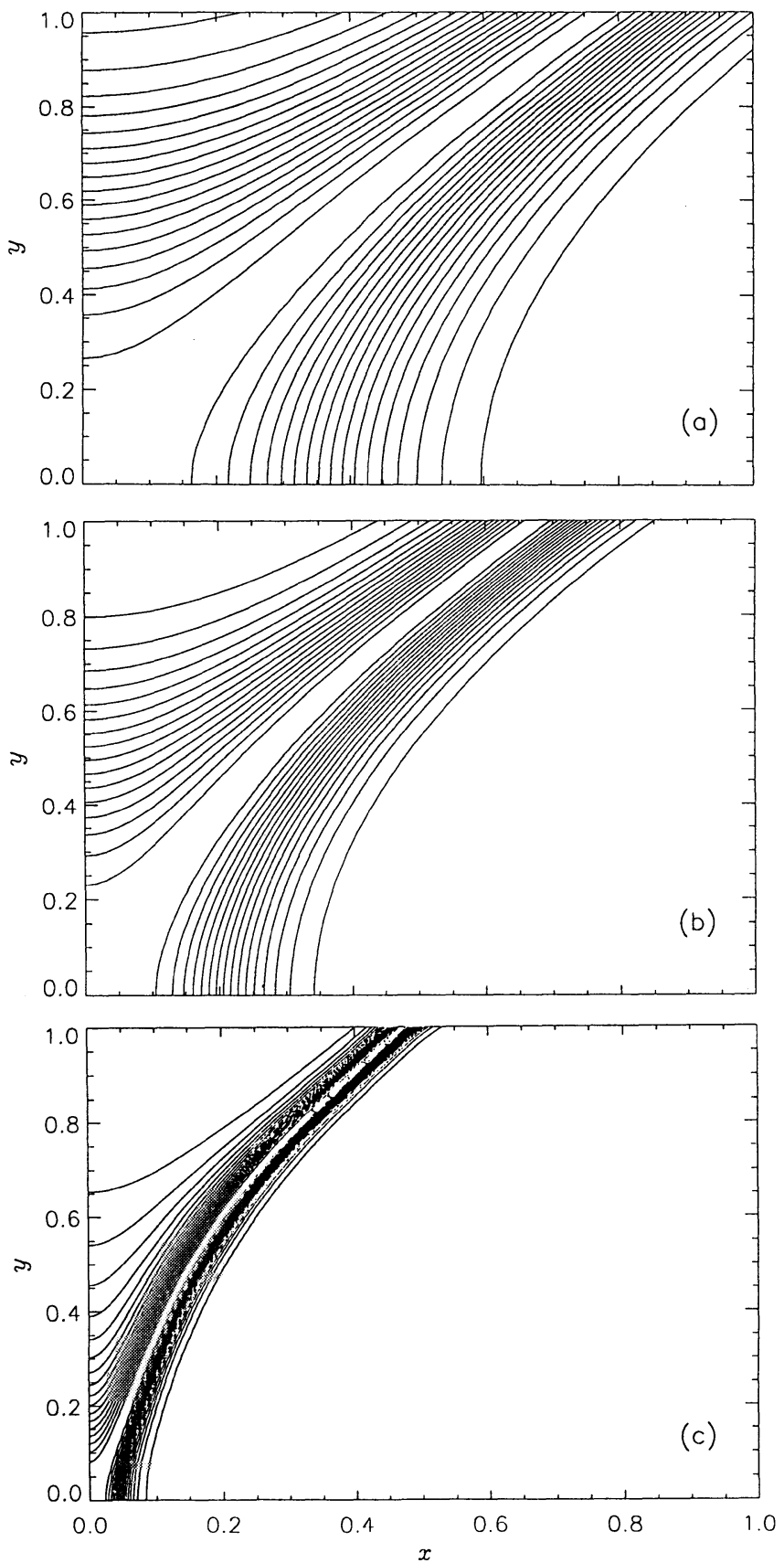

Fig. 6.-Contour plots of current density $(\eta=1.0,0.5,0.1$ in diagrams $(a)$, $(b)$, and (c), respectively). The rapid collapse toward a quasi one-dimensional current sheet is immediately apparant.

However, we take the view that if fast dissipation is possible for the resistive system as is, then fast reconnection should persist when local effects allowing greater freedom in the flow topology are introduced. This view contrasts with Priest et al. (1994) who modify the resistive system on the grounds that solutions which disqualify motion across the separatrices are qualitatively unlike historical reconnection models.

\section{DISCUSSION AND CONCLUSIONS}

We have considered the incompressible, resistive system of traditional steady state theory. Under the restriction of subAlfvénic flow speeds, a global treatment is possible which allows the velocity stream function to be expressed analytically as a functional of the magnetic field structure. This treatment shows that

1. The magnetic field lines are characteristics of the velocity stream function along which the pressure and current density are constant. Ridges of peak current contiguous with the separatrices extend uniformly from the origin to the outer boundary.

2. All separatrix flows vanish; strong shear flows occur parallel to the separatrices.

3. The neutral point is a saddle point of the pressure profile: it is a local maximum in the direction of the inflow but a local minimum along the outflow.

It should be stressed that these properties can only be undone by modifying the system, say by assuming nonuniform resistivities (Yan et al. 1992), or by including viscous damping (Priest et al. 1994). Either way, classical "solutions" which assume separatrix flows and current structures localized to the neutral point are misleading as models of the incompressible resistive system.

Turning to the possibility of fast magnetic energy dissipation, we have shown that the constraint of vanishing separatrix flows is still compatible with fast reconnective solutions. In particular, the models of $\S 3$ provide an explicit demonstration that "open" topologies allow fast reconnection to persist under conditions that lead to stalling in "closed" or periodic geometries (Craig, Henton, \& Rickard 1993; Craig \& McClymont 1993). In nonlinear applications the reconnection rate is maintained against reductions in plasma resistivity by the increasing amplitude, but decreasing width, of the separatrix current layer. By way of constrast, the linear models are characterized by current "spikes" at the origin whose are a contracts uniformly with resistivity.

Finally we mention that, although we have explored only a limited class of nonlinear models, a cursory glance at reconnection simulations reveals many of the features predicted by our analysis. The tendency for the strong separatrix shear flows is obscured by pseudoviscous effects required for numerical stability, but the presence of global current structures extending uniformly along the separatrices is unmistakable in both steady state and dynamic computations of compressible plasmas (e.g., Sato \& Hayashi 1979; Biskamp 1986). Convincing nonlinear simulations of fast reconnection have yet to be achieved, but our analysis suggests that narrow strips of high current $\left[O\left(\eta^{-1}\right)\right]$ contiguous with the separatrices of the field should be a signature for classes of fast reconnective models.

We are pleased to acknowledge helpful discussions with A. D. Sneyd. 
Biskamp, D. 1986, Phys. Fluids, 29, 1520

Biskamp, D. \& Welter, H. 1980, Phys. Rev. Lett. 44, 1069

I $\rightarrow 1$ Craig, I. J. D., Henton, S. M., \& Rickard, G. J. 1993, A\&A, 267, L39

Craig, I. J. D., \& McClymont, A. N. 1991, ApJ, 371, L41 1993, ApJ, 405, 207

Craig, I. J. D., \& Rickard, G. J. 1994, A\&A, 287, 261

Craig, I. J. D., \& Watson, P. G. 1992, ApJ, 393, 385

DeLuca, E. E., \& Craig, I. J. D. 1992, ApJ, 390, 679

Forbes, T. G., \& Priest, E. R. 1987, Rev. Geophys., 25, 1583

Hassam, A. B. 1991, ApJ, 399, 159

Petschek, H. E. 1964, in Proc. AAS-NASA Symp. Physics of Solar Flares (NASA SP-50), 425

\section{REFERENCES}

Priest, E. R., \& Forbes, T. G. 1986, J. Geophys. Res., 91, 5579

Priest, E. R., Titov, V. S., Vekstein, G. E., \& Rickard, G. J. 1994, preprint

Rickard, G. R., \& Craig, I. J. D. 1993, Phys. Fluid, B, 5, 956

Sato, T., \& Hayashi, T. 1979, Phys. Fluids, 22, 1189

Vasyliunas, V. M. 1975, Rev. Geophys. Space Sci., 13, 303

Yan, M., Lee, L. C., \& Priest, E. R. 1992, J. Geophys. Res., 97, 8277 